\title{
HIV Viral Dynamic under Treatment with Intracellular Delay and Virus Decay as Interactive Parameters
}

\author{
R. A. C. PRATA ${ }^{1}$, R. M. JAFELICE ${ }^{2}$, V. M. CABRAL ${ }^{3}$, \\ F. S. PEDRO ${ }^{4 *}$ and L. C. BARROS ${ }^{5}$
}

Received on June 10, 2019 / Accepted on January 25, 2021

\begin{abstract}
Treatment with antiviral drugs for human immunodeficiency virus type 1 (HIV-1) infection causes a rapid reduction in plasma viral load. Viral decline occurs in several stages and provides information on important kinetic constants of virus replication in vivo and pharmacodynamic properties. We present a mathematical model that not only considers the intracellular phase of the viral life cycle, defined as the time between the infection of a cell and the production of new viral particles, but we also consider that this parameter together with the virus decay are interactive fuzzy numbers.
\end{abstract}

Keywords: joint possibility distribution, interactive fuzzy numbers, HIV model.

\section{INTRODUCTION}

HIV-1 infection and immunodeficiency, which characterizes AIDS, occurs in three stages. After HIV-1 infection, the amount of virus in the blood increases to high levels and symptoms begin to appear. After a few weeks, or in some cases months, the viral load stabilizes at lower values. It then begins a long period of clinical latency, in which the virus persists at a relatively stable level and the CD4+ T-cell count drops gradually. This period is when the patient is asymptomatic and can last for years. In the final stages of the disease, the viral load increases rapidly and the CD4+ T-cell count decreases. Thus, the immune system does not provide protection against opportunistic infections leading to death.

\footnotetext{
*Corresponding author: Francielle Santo Pedro - E-mail: fran.stopedro@gmail.com

${ }^{1}$ Department of Mathematics, Federal University of Amazonas, Manaus (UFAM), Brazil - E-mail: praroberto@gmail.com https://orcid.org/0000-0001-7578-1014

2 Faculty of Mathematics, Federal University of Uberlândia (UFU), Uberlândia, Brazil _ E-mail: rmotta@ufu.br https://orcid.org/0000-0001-8489-3974

${ }^{3}$ Department of Mathematics, Federal University of Amazonas (UFAM), Manaus, Brazil - E-mail: vmcabral@gmail.com https://orcid.org/0000-0002-3130-2752

${ }^{4}$ Multidisciplinary Department, Federal University of São Paulo (UNIFESP), Osasco, Brazil. - E-mail: fran.stopedro@gmail.com https://orcid.org/0000-0001-5177-563X

${ }^{5}$ Department of Applied Mathematics, University of Campinas, Campinas (UNICAMP), Brazil - E-mail: laeciob@unicamp.br https://orcid.org/0000-0003-4322-5974
} 
One of the challenge is to understand the processes that occur in this long asymptomatic period of the disease. Viral load measurements alone do not provide any information since they hardly change.

By disturbing this latent state with potent antiretroviral drugs, important information about underlying dynamic processes was obtained. These studies demonstrate high viral turnover rates for both the free virus and the infected cells. In addition, they explain the rapid development of drug resistance and have influenced strategies for the treatment of HIV-1 infection with combinations of antiretroviral drugs [11].

Herz et al. [8] used a simple model where the delays that occur between administration of drugs and the observed decline in viral load are considered fixed, and include a term for the sum of these delays. They came to the conclusion that the inclusion of an intracellular delay would substantially shorten the estimate of the half-life of the free virus.

In this manuscript, we will study the viral dynamics from the point of view of possibility distribution. The justification for such use comes from the fact that data from in vitro viral infection studies present many uncertainties, but they clearly indicate that there is a delay between the initial infection and the production of new viruses. In addition, Herz et al. [8] state that estimates for the rate of viral concentration decline and intracellular delay are positively correlated.

\section{MATHEMATICAL BACKGROUND}

A fuzzy subset $A$ of $\mathbb{R}$ is characterized by a membership function $\mu_{A}: \mathbb{R} \rightarrow[0,1]$, where $\mu_{A}(x)$ means the degree that $x$ belongs to $A$. The $\alpha$-levels of $A$ is defined by the classical subsets

$$
\begin{aligned}
& {[A]^{\alpha}=\left\{x \in \mathbb{R}: \mu_{A}(x) \geq \alpha\right\} \text { for } 0<\alpha \leq 1 \text { and }} \\
& {[A]^{0}=\overline{\left\{x \in \mathbb{R}: \mu_{A}(x)>0\right\}} \text { for } \alpha=0 .}
\end{aligned}
$$

For $\alpha=1,[A]^{1}$ is called the core of $A$.

The fuzzy subset $A$ is called a fuzzy number if all their $\alpha$-levels are closed and nonempty intervals of $\mathbb{R}$ and the support of $A, \operatorname{supp}(A)=\left\{x \in \mathbb{R}: \mu_{A}(x)>0\right\}$, is limited. The family of fuzzy number is denoted by $\mathbb{R}_{\mathscr{F}}$ whilst the family of fuzzy number with nonempty compact and convex $\alpha$-levels is denoted by $\mathscr{F}(\mathbb{R})$.

A triangular fuzzy number is denoted by the triple $(a ; b ; c)$, where $a \leq b \leq c$, and its $\alpha$-levels are given by [1]

$$
[a+\alpha(b-a), c-\alpha(c-b)], \forall \alpha \in[0,1]
$$

A joint possibility distribution in $\mathbb{R}^{n}$ is a fuzzy subset $J$ of $\mathbb{R}^{n}$ with membership function $\mu_{J}$ : $\mathbb{R}^{n} \rightarrow[0,1]$, such that $\mu_{J}\left(x_{0}\right)=1$ for some $x_{0} \in \mathbb{R}^{n}$. The family of possibility distributions in $\mathbb{R}^{n}$ is denoted by $\mathscr{F}_{J}\left(\mathbb{R}^{n}\right)$.

Let $A$ and $B$ be fuzzy numbers. According to [6], $J \in \mathscr{F}\left(\mathbb{R}^{2}\right)$ is a joint possibility distribution of $A$ and $B$ if

$$
\max _{x_{2}} \mu_{J}\left(x_{1}, x_{2}\right)=\mu_{A}\left(x_{1}\right) \text { and } \max _{x_{1}} \mu_{J}\left(x_{1}, x_{2}\right)=\mu_{B}\left(x_{2}\right)
$$


for all $x_{1}, x_{2} \in \mathbb{R}$. Moreover, $\mu_{A}$ and $\mu_{B}$ are called marginal possibility distribution of $J$.

The fuzzy numbers $A$ and $B$ are said to be non-interactive if and only if the following holds

$$
\mu_{J}\left(x_{1}, x_{2}\right)=\min \left\{\mu_{A}\left(x_{1}\right), \mu_{B}\left(x_{2}\right)\right\}, \text { and, in this case }[J]^{\alpha}=[A]^{\alpha} \times[B]^{\alpha},
$$

for all $\left(x_{1}, x_{2}\right) \in \mathbb{R}^{2}$ and $\alpha \in[0,1]$. Otherwise they are said to be interactive.

Let $J$ be a joint possibility distribution of $A$ and $B$, and $f: \mathbb{R}^{2} \longrightarrow \mathbb{R}$ a continuous function. According to $[4,6]$, the extension of $f$ via $J$ is a function $f_{J}$, whose membership function is given by

$$
\mu_{f_{J(A, B)}}(y)=\left\{\begin{array}{ccc}
\sup _{\left(x_{1}, x_{2}\right) \in f^{-1}(y)} \mu_{J}\left(x_{1}, x_{2}\right), & \text { if } & f^{-1}(y) \neq \emptyset \\
0, & \text { if } & f^{-1}(y)=\emptyset
\end{array},\right.
$$

where $f^{-1}(y)=\left\{\left(x_{1}, x_{2}\right) \in \mathbb{R}^{2}: f\left(x_{1}, x_{2}\right)=y\right\}$.

Theorem 2.1. [4, 6] If $A, B \in \mathbb{R}_{\mathscr{F}}, J$ their joint possibility distribution and $f: \mathbb{R}^{2} \longrightarrow \mathbb{R} a$ continuous function, then

$$
\left[f_{J}(A, B)\right]^{\alpha}=f\left([J]^{\alpha}\right), \text { for all } \alpha \in[0,1] .
$$

It is worth noting that when $\mu_{J}\left(x_{1}, x_{2}\right)=\min \left\{\mu_{A}\left(x_{1}\right), \mu_{B}\left(x_{2}\right)\right\}$, then we have the Zadeh extension. In this case, $\left[f_{J}(A, B)\right]^{\alpha}=f\left([A]^{\alpha} \times[B]^{\alpha}\right)$.

\section{$3 \quad F$-INTERACTIVE FUZZY NUMBERS}

In this section we present a study on $f$-interactive fuzzy numbers using the concept of possibility distribution.

According to [4] the fuzzy numbers $A$ and $B$ are called $f$-interactive if

$$
\mu_{B}(y)=\left\{\begin{array}{ccc}
\sup _{y=f(z)} \mu_{A}(z) & \text { if } & f^{-1}(y) \neq \emptyset \\
0 & \text { if } & f^{-1}(y)=\emptyset
\end{array} .\right.
$$

Notice that when $A$ and $B$ are $f$-interactive, then $B$ is coincide with Zadeh's extension principle of the function $f$ evaluated at the fuzzy number $A$, that is, $B=f(A)$. Moreover, if $f$ is invertible, then $A=f^{-1}(B)$. In this case,

$$
[J]^{\alpha}=\left\{(x, f(x)) \in \mathbb{R}^{2}: x \in[A]^{\alpha}\right\}=\left\{\left(f^{-1}(y), y\right) \in \mathbb{R}^{2}: y \in[B]^{\alpha}\right\} .
$$

Also, if $f$ is continuous function, then $[B]^{\alpha}=f\left([A]^{\alpha}\right)$.

Next we will define the operations between $f$-interactive fuzzy numbers.

Let $A, B$ be $f$-interactive fuzzy numbers. According to $[4,13]$, the arithmetic operation $B *_{f} A$ is obtained by

$$
\mu_{B *_{f} A}(z)=\left\{\begin{array}{ccc}
\sup _{(x, y) \in \phi^{-1}(z)} \mu_{J}(x, y) & \text { if } & \phi^{-1}(z) \neq \emptyset \\
0 & \text { if } & \phi^{-1}(z)=\emptyset
\end{array}\right.
$$


where $\phi^{-1}(z)=\{x * y=z: y=f(x)\}$ and $* \in\{+,-, \cdot, \div\}$.

Notice that the arithmetic operations between two $f$-interactive fuzzy numbers are given as restrictions of the traditional operators on the curve $\left\{(x, y) \in \mathbb{R}^{2}: y=f(x)\right\}$.

According to Theorem 2.1, for all $\alpha \in[0,1]$, the four operations of $f$-interactive fuzzy numbers are given by

- $\left[B+{ }_{f} A\right]^{\alpha}=\left\{(f(x)+x) \in \mathbb{R}: x \in[A]^{\alpha}\right\}$

- $\left[B-{ }_{f} A\right]^{\alpha}=\left\{(f(x)-x) \in \mathbb{R}: x \in[A]^{\alpha}\right\} ;$

- $\left[B \cdot{ }_{f} A\right]^{\alpha}=\left\{(x f(x)) \in \mathbb{R}: x \in[A]^{\alpha}\right\}$;

- $\left[B \div{ }_{f} A\right]^{\alpha}=\left\{(f(x) / x) \in \mathbb{R}: x \in[A]^{\alpha}\right\}$.

Following, aiming at the dynamic model that will be studied in Section 5, we present a particular case of $f$-interactive fuzzy numbers.

Consider $f$ as being a hyperbolic function $f: \mathbb{R} \rightarrow \mathbb{R}$, given by $f(x)=\frac{q}{x}+r, x \neq 0$. Two fuzzy numbers $A$ and $B$ are said to be hyperbolically interactive, if there exist $q \neq 0, r \in \mathbb{R}$, such that its joint possibility distribution $J$ is given by

$$
\begin{aligned}
\mu_{J}(x, y) & =\mu_{A}(x) \mathscr{X}_{\left\{\frac{q}{x}+r=y\right\}}(x, y) \\
& =\mu_{B}(y) \mathscr{X}_{\left\{\frac{q}{x}+r=y\right\}}(x, y)
\end{aligned}
$$

where $\mathscr{X}_{\left\{\frac{q}{x}+r=y\right\}}$ is the characteristic function of line $\left\{(x, y) \in \mathbb{R}^{2}: \frac{q}{x}+r=y\right\}$. In this case, $[B]^{\alpha}=\left\{\frac{q}{x}+r: x \in[A]^{\alpha}\right\}, 0 \notin[A]^{0}$.

For all $\alpha \in[0,1]$, the four arithmetic operations of hyperbolically interactive fuzzy numbers are given by

- $\left[B+{ }_{f} A\right]^{\alpha}=\left\{\left(\frac{q+x^{2}}{x}+r\right) \in \mathbb{R}: x \in[A]^{\alpha}\right\}, 0 \notin[A]^{0} ;$

- $\left[B-{ }_{f} A\right]^{\alpha}=\left\{\left(\frac{q-x^{2}}{x}+r\right) \in \mathbb{R}: x \in[A]^{\alpha}\right\}, 0 \notin[A]^{0}$;

- $\left[B \cdot{ }_{f} A\right]^{\alpha}=\left\{(q+x r) \in \mathbb{R}: x \in[A]^{\alpha}\right\}, 0 \notin[A]^{0}$;

- $\left[B \div{ }_{f} A\right]^{\alpha}=\left\{\left(\frac{q+x r}{x^{2}}\right) \in \mathbb{R}: x \in[A]^{\alpha}\right\}, 0 \notin[A]^{0}$.

It is worth stressing that if we take $r=0$ and $q=1$, we have $\left[B \cdot_{f} A\right]^{\alpha}=1$, for all $\alpha \in[0,1]$.

\section{MEASURES OF CENTRAL TENDENCY AND MEASURES OF DISPERSION FOR $F$-INTERACTIVE FUZZY NUMBERS}

Next, we define central value, expected value, measure of interactivity, variance, covariance and index of interactivity via possibility distribution as it was done in [7]. 
A function $\omega:[0,1] \rightarrow \mathbb{R}$ is called a weighting function if it is non-negative, monotone increasing and normalized over the unit interval $[0,1]$, that is,

$$
\int_{0}^{1} \omega(s) d s=1
$$

Let $p: \mathbb{R}^{2} \rightarrow \mathbb{R}$ be an integrable function and $J$ be the joint possibility distributions in $\mathbb{R}^{2}$. The central value of $p$ on $[J]^{\alpha}$ is given by

$$
\mathscr{C}_{[J]_{\alpha}}(p)=\frac{1}{\int_{[J]_{\alpha}} d x} \int_{[J]_{\alpha}} p(x) d x .
$$

The expected value $p$ on $J$, with respect to a weighting function $\omega$, is given by

$$
E_{\omega}(p ; J)=\int_{0}^{1} \mathscr{C}_{[J]_{\alpha}}(p) \omega(\alpha) d \alpha .
$$

Let $J$ be a joint possibility distribution in $\mathbb{R}^{2}$ with marginal possibility distributions $A$ and $B$. The measure of interactivity between the $\alpha$-levels $A$ and $B$ (with respect to $[J]_{\alpha}$ ) is defined by

$$
\begin{aligned}
& I\left([A]^{\alpha},[B]^{\alpha}\right)=\frac{1}{\int_{[J]^{\alpha}} d x d y} \int_{[J]^{\alpha}} x y d x d y- \\
& -\left(\frac{1}{\int_{[A]^{\alpha}} d x} \int_{[A]^{\alpha}} x d x\right)\left(\frac{1}{\int_{[B]^{\alpha}} d y} \int_{[B]^{\alpha}} y d y\right) .
\end{aligned}
$$

Let $J$ be a joint possibility distribution in $\mathbb{R}^{2}$, and $A, B \in \mathbb{R}_{\mathscr{F}}$ be its marginal possibility distribution. The measure of interactivity between $A$ and $B$ (with respect to their joint possibility distribution $J$ and weighting function $\omega$ ) is defined by

$$
I_{\omega}(A, B)=\int_{0}^{1} I\left([A]^{\alpha},[B]^{\alpha}\right) \omega(\alpha) d \alpha .
$$

It is worth to notice that, when one considers the uniform distribution, the Equation (4.2) reflects the covariance between the characteristic functions of $[A]_{\alpha}$ and $[B]_{\alpha}$. According to [13], if $A, B \in$ $\mathbb{R}_{\mathscr{F}} \backslash \mathbb{R}$ are $f$-interactive, where $f$ is a monotone continuous function and $B=f(A)$, then (4.3) becomes $I_{\omega}(A, B)=$

$$
\int_{0}^{1}\left(\frac{1}{a_{\alpha}^{+}-a_{\alpha}^{-}} \int_{a_{\alpha}^{-}}^{a_{\alpha}^{+}} x f(x) d x-\frac{\left(a_{\alpha}^{+}+a_{\alpha}^{-}\right)\left(b_{\alpha}^{+}+b_{\alpha}^{-}\right)}{4}\right) \omega(\alpha) d \alpha .
$$

If $[A]^{\alpha}=\left[a_{1}^{\alpha}, a_{2}^{\alpha}\right]$ and $[B]^{\alpha}=\left[b_{1}^{\alpha}, b_{2}^{\alpha}\right]$, for all $\alpha \in[0,1]$, and $\operatorname{supp}(A) \in \mathbb{R}^{+}$with $A$ and $B$ being hyperbolically interactive, that is,

$$
\begin{gathered}
{[B]^{\alpha}=f\left([A]^{\alpha}\right)=\frac{q}{[A]^{\alpha}}+r \Leftrightarrow} \\
{\left[b_{1}^{\alpha}, b_{2}^{\alpha}\right]= \begin{cases}{\left[\frac{q}{a_{\alpha}^{-}}+r, \frac{q}{a_{\alpha}^{+}}+r\right.} \\
\left.\frac{q}{a_{\alpha}^{+}}+r, \frac{q}{a_{\alpha}^{-}}+r\right], & q \geq 0\end{cases} }
\end{gathered}
$$


then, by (4.4) and (4.5), it holds that

$$
I_{\omega}(A, B)=\frac{ \pm q}{4} \int_{0}^{1} \frac{\left(a_{1}^{\alpha}-a_{2}^{\alpha}\right)^{2}}{a_{1}^{\alpha} a_{2}^{\alpha}} \omega(\alpha) d \alpha,
$$

where the sign is positive if $A$ and $B$ are positive hyperbolically interactive and negative if $A$ and $B$ are negative hyperbolically interactive.

In the next section, we present the HIV dynamic where the delay and death rate of the virus are hyperbolically interactive .

\section{HIV VIRAL DYNAMIC WITH DELAY AND DEATH RATE OF THE VIRUS HYPERBOLICALLY INTERACTIVE}

In recent years, many clinical studies with drug therapy combined with mathematical modeling have been done in order to better understand the human immunodeficiency virus type 1 (HIV-1) infection. From these studies it was possible to estimate important kinetic parameters of virus replication in vivo associated with the dynamics of HIV-1 infected individuals $[8,9,12]$.

The model that describes the virus dynamics in individuals who are in antiretroviral therapy is given by [12]

$$
\left\{\begin{array}{rl}
\frac{d x(t)}{d t} & =\lambda-c x(t)-\beta(t) x(t) v(t) \\
\frac{d y(t)}{d t} & =\beta(t) x(t) v(t)-a y(t) \\
\frac{d v(t)}{d t} & =k(t) y(t)-u v(t)
\end{array},\right.
$$

where

- $x(t)$ is the population of the cell uninfected;

- $y(t)$ is the population of the cell infected that produce virus;

- $v(t)$ is the population of the virus;

- $\lambda$ is the influx blood;

- $c$ is the death rate of the cells uninfected;

- $\beta(t) x(t) v(t)$ is the rate of the produce of the cells infected;

- $u$ is the rate of the decline of the virus concentration;

- $a$ is the death rate of the cells infected;

- $k(t)$ is the rate of the produce free virus particles. 
An accurate estimation of half-life is not always possible since sampling in the early stages of treatment is not frequent enough. Thus, considering the delay in model (5.1) is of crucial importance in estimating the half-life [12].

When considering biologically realistic differences in the state of cellular activation, metabolism, cell position in the cell cycle, pre-existing reserves of nucleotides and other precursors required for the production of new virus, along with genetic variation in the viral population, variation in delay of infection become almost a certainty [11].

Whenever a cell is infected, it takes a time $\tau_{\text {int }}$ to produce the particles of the virus and after application of any anti-viral drug there is a delay in pharmacological effect due to the time needed for the absorption, distribution, and penetration into the target cells [9]. Let $\tau$ be the delay the virus produces behind the infection of a cell. This implies that the recruitment of virus-producing cells, at time $\mathrm{t}$, is given by the density of cells that were newly infected at time $t-\tau$ and are still alive at time $t$ [12]. We will assume that $\tau=\tau_{\text {int }}+\tau_{\text {farm }}$.

According to [12], the system (5.1) with delay $\tau$ is given by

$$
\begin{aligned}
\frac{d x(t)}{d t} & =\lambda-c x(t)-\beta(t) x(t) v(t) \\
\frac{d y(t)}{d t} & =\beta(t-\tau) x(t-\tau) v(t-\tau) e^{-\widetilde{a} \tau}-a y(t) \\
\frac{d v(t)}{d t} & =k(t) y(t)-u v(t)
\end{aligned}
$$

where

- $\tilde{a}$ is the death rate of the cells uninfected, but not yet produce virus;

- $e^{-\widetilde{a} \tau}$ is the probability of survival of infected cells from the time $t-\tau$ to the time $t$. Generally, the probability of survival is given by function $\delta(\tau)$ with $0 \leq \delta(\tau) \leq 1$.

Notice that, in the model in question, the population of cells uninfected, infected virus-producing cells, and virus free are at a steady-state level before treatment is established. Thus, it is possible obtaining simple analytic solutions of $(5.3)[8,12]$. The nontrivial solution for the steady state is given by

$$
\begin{aligned}
x_{0} & =\frac{a u}{\beta k} e^{\widetilde{a} \tau} \\
y_{0} & =\frac{\lambda}{a} e^{\widetilde{a} \tau}-\frac{u c}{\beta k} \\
v_{0} & =\frac{k y_{0}}{u},
\end{aligned}
$$

where $\beta$ and $k$ are constant of pretreatment rates. 


\subsection{Protease inhibitor therapy}

By including treatment with HIV protease inhibitors, the production of new infectious virus $\left(v_{I}\right)$ from already infected cells is blocked. Thus, only noninfectious virus is generated. Allowing to previously released infectious virus decays but continues to infect cells $[8,12]$.

According to [8], Equation 5.4 still describes the dynamics of the total free virus. In spite of that infectious virus is not produced at $t>0$ and decline according to

$$
\frac{d v_{I}(t)}{d t}=-u v_{I}(t)
$$

Equations 5.2 and 5.3 remain valid replacing $v$ by $v_{I}$.

As in [8], under the scale of time in consideration, we assumed that the population of uninfected cells remains constant, that is, $x(t)=x_{0}$. From $x(t)=x_{0}$ and $v_{I}(t)$ declining exponentially, we obtain that solution of (5.3) is given by

$$
y(t)=\frac{y_{0}}{a-u}\left[a e^{-u(t-\tau)}-u e^{-a(t-\tau)}\right] \text { for } t>\tau .
$$

From (5.4), the time of evolution of the free virus is given by $v(t)=v_{0}$ for $0<t \leq \tau$, and

$$
v(t)=v_{0} e^{-u(t-\tau)}+\frac{u v_{0}}{a-u}\left[\frac{u\left(e^{-a(t-\tau)}-e^{-u(t-\tau)}\right)}{a-u}+a(t-\tau) e^{-u(t-\tau)}\right]
$$

for $t>\tau$.

Equation 5.5 provides a single and consistent description of short-time HIV dynamics, including the effect of intracellular delay.

Herz et al. [8], states that precise estimates of the pharmacological delay, the intracellular delay, and free virus half-life are impossible with current data on plasma virus decay. By experimental data, they concluded that $u$ and $\tau$ are uncertain and are positively correlated. Thus, in the average viral generation time

$$
T=\tau+\frac{1}{u}+\frac{1}{a}
$$

these uncertainties nullify each other partially.

The viral decline curves can be divided into three stages: an initial phase during which viral density remains relatively constant, a transition phase during which it begins to decrease, and a final phase during which virus declines exponentially. The shape of the decline during the first two phases has been referred as the viral "shoulder" $[8,11,12]$.

In vitro data are fraught with uncertainties and some authors assume that the delay is given by a probability distribution $[8,11,12]$.

Jafelice et al. [9] have modeled the relationship between the rate of decline in the plasma virus concentration $u$ and the intracellular delay $\tau$ via Takagi-Sugeno inference (TSI). After, the HIV decay curve for (5.5) was determined by Zadeh's Extension Principle. That is, they have considered $\tau$ and $u$ non-interactive fuzzy numbers. As in [5], we go to explore the interaction between $\tau$ 
and $u$ by hyperbolically fuzzy numbers and, more ahead explore the autoregressive fuzzy process of the virus population.

\section{FUZZY SOLUTION FOR FREE VIRUS POPULATION WITH DELAY AND VIRUS DEATH RATE HYPERBOLICALLY FUZZY INTERACTIVE}

As Massad et al. states in [10], mathematical models for biological phenomena are normally fraught with uncertainties, in both the state variables and the parameters of the model's equations.

From equation (5.6) we propose the study of the decline of the viral load after the beginning of protease inhibitor therapy that considers the intracellular and pharmacological delay via hyperbolically fuzzy numbers. Moreover, we will consider that the delay time $\tau$ is modeled by a fuzzy number.

According to [8], the average viral generation time $(T)$ varies between $T=2.5$ and $T=3.1$ days, and $\tau$ assumes the values of 0.08 day (approximately $2 \mathrm{~h}$ ), 0.5 day (12 h), and 1 day (24 h).

Let us consider $T=3$ and $a=0.5 /$ day, as in [9]. Thus, from (5.6), we have

$$
\tau=1-\frac{1}{u} \Leftrightarrow u=\frac{1}{1-\tau},
$$

where $\tau$ is modeled by triangular fuzzy number given by

$$
\Gamma=(0.08 ; 0.5 ; 0.88)
$$

equivalently,

$$
[\Gamma]^{\alpha}=[0.08+0.42 \alpha, 0.88-0.38 \alpha]
$$

By (6.1) and (6.2), the rate of the decline of the virus concentration $u$ is given by the fuzzy number $U$ as follow

$$
[U]^{\alpha}=\frac{1}{1-[\Gamma]^{\alpha}}=\left[\frac{1}{0.92-0.42 \alpha}, \frac{1}{0.12+0.38 \alpha}\right],
$$

by (3.1), the joint possibility distribution $J$ between $\Gamma$ and $U$, is given by

$$
\mu_{J}(u, \tau)=\mu_{\Gamma}(\tau) \mathscr{X}_{\left\{1-\frac{1}{u}=\tau\right\}}(u, \tau)
$$

equivalently,

$$
[J]^{\alpha}=\left\{\left(u, 1-\frac{1}{u}\right) ; u=(1-s)\left[\frac{1}{0.92-0.42 \alpha}\right]+s\left[\frac{1}{0.12+0.38 \alpha}\right], s \in[0,1]\right\} .
$$



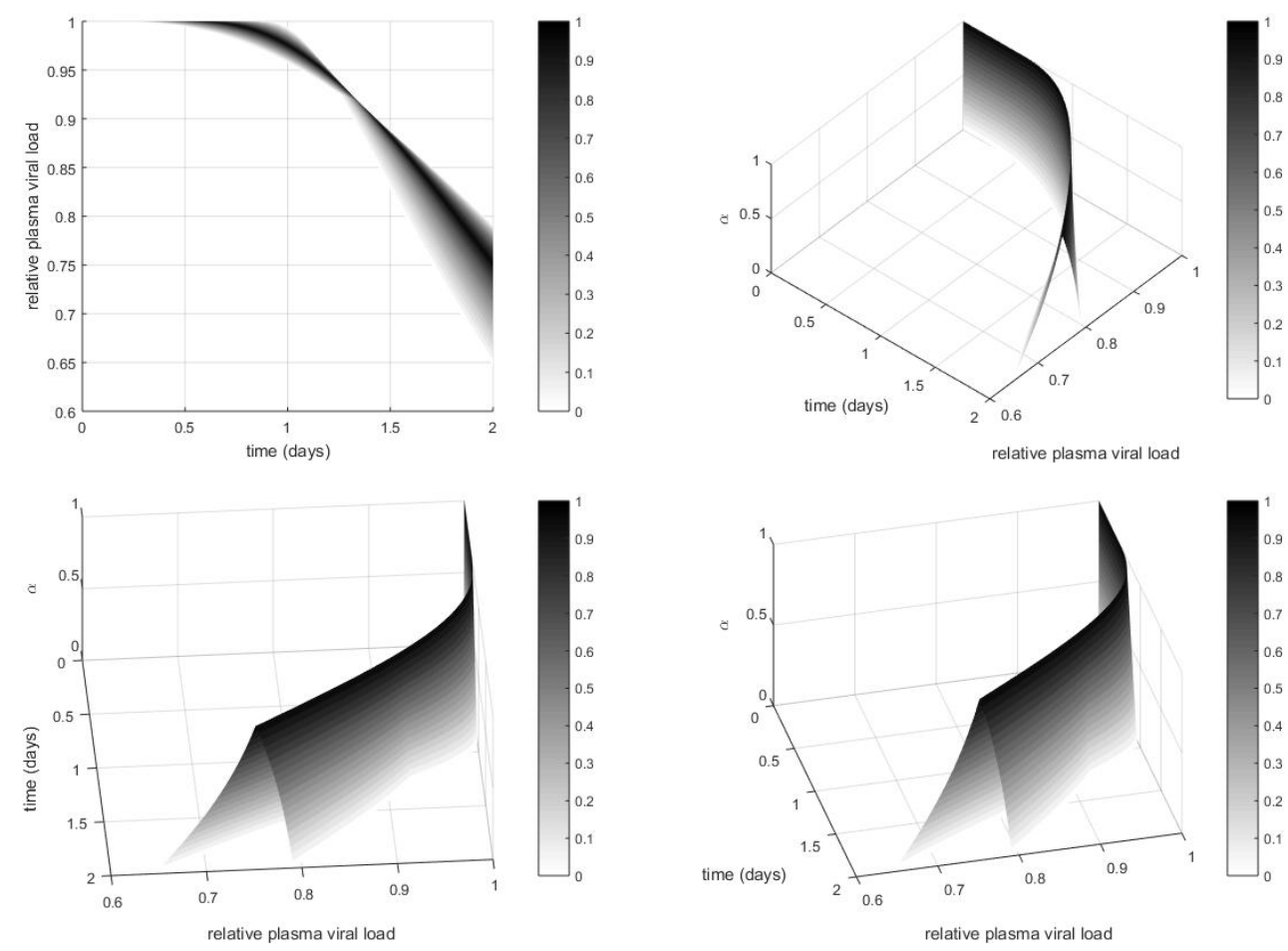

Figure 1: 3D graphical representation of fuzzy plasma viral load population (Equation 6.4). The graph is in logarithmic scale. The gray lines represent the $\alpha$-cuts of the $v_{t}(\tau, u)$. The endpoints for $\alpha$, varying from 0 to 1 , are represented respectively by the gray-scale lines varying from white to black.

Now, for each $t>0.08$, the fuzzy solution for the viral load is obtained by Theorem 2.1 and Equation 5.5 as follows $\left[\left(v_{t}\right)_{J}(U, \Gamma)\right]^{\alpha}=\left(v_{t}\right)\left([J]^{\alpha}\right)=\left\{v_{t}(u, \tau) ;(u, \tau) \in[J]^{\alpha}\right\}=$

$$
\begin{aligned}
& \left\{v_{0} e^{-u(t-\tau)}+\frac{u v_{0}}{0.5-u}\left[\frac{u\left(e^{-0.5(t-\tau)}-e^{-u(t-\tau)}\right)}{0.5-u}+0.5(t-\tau) e^{-u(t-\tau)}\right] ; \tau=1-\frac{1}{u}\right\}= \\
& \left\{v_{0} e^{-u\left(t+\frac{1}{u}-1\right)}+\frac{u v_{0}}{0.5-u}\left[\frac{u\left(e^{-0.5\left(t+\frac{1}{u}-1\right)}-e^{-u\left(t+\frac{1}{u}-1\right)}\right)}{0.5-u}+0.5\left(t+\frac{1}{u}-1\right) e^{-u\left(t+\frac{1}{u}-1\right)}\right]\right. \\
& \left.; u \in[U]^{\alpha}\right\} .
\end{aligned}
$$

Figure 1 shows the graphic of fuzzy solution, $v_{t}(\tau, u)$, of the fuzzy model (6.4). The solution, $v_{t}(\tau, u)$, describes the short-time HIV dynamics that explicitly incorporates intracellular delays. 


\subsection{Effect of interactivity between $U$ and $\Gamma$ on the behavior of plasma viral load population}

In this subsection, we study the effect of interactivity between $U$ and $\Gamma$ on the behavior of the plasma viral load population and also on the curve obtained of the defuzzified solutions, which was obtained via the centroid defuzzification method [1]. First, we analyze the influence of interactivity between $U$ and $\Gamma$ on the fuzzy curve (6.4) via the interactivity measure, and then its effect on the defuzzified curve.

The defuzzified solution is a deterministic curve obtained by (4.1), that is, it is the expected value of $v_{t}(\tau, u)$ where the weighting function is given by beta distribution. The choice makes sense, since it is frequently used to model objects belonging to the interval $(0,1)$ [2]. Moreover, for $a>b$, the beta function is monotonic and increasing, and in this way suitable for the weighting function as stated in Section 4. From now on, unless we say the opposite, we will consider the weighting function given by the beta distribution

$$
\omega(\alpha)=\frac{\alpha^{a-1}(1-\alpha)^{b-1}}{\int_{0}^{1} \alpha^{a-1}(1-\alpha)^{b-1} d \alpha},
$$

where the beta function is $B(a, b)=\int_{0}^{1} \alpha^{a-1}(1-\alpha)^{b-1} d \alpha$.

- The influence of interactivity between $U$ and $\Gamma$ on the fuzzy plasma viral load population.

The hyperbolic function is given by $\tau=1-\frac{1}{u}$, thus, replacing $q=-1, r=1$, and the $\alpha$-levels of $\Gamma$ (given by (6.2)) in (4.6), we obtain

$$
\begin{aligned}
I_{\omega}(U, \Gamma) & =\frac{1}{4} \int_{0}^{1} \frac{(0.8 \alpha-0.8)^{2}}{(0.92-0.42 \alpha)(0.12+0.38 \alpha)} \omega(\alpha) d \alpha \\
& =\frac{1}{4} \int_{0}^{1} \frac{(0.8 \alpha-0.8)^{2} \alpha^{(a-1)}(1-\alpha)^{b-1}}{(0.92-0.42 \alpha)(0.12+0.38 \alpha) B(a, b)} d \alpha .
\end{aligned}
$$

We compute (6.6) for several values of beta function as one can see in Table 1.

Table 1: The measure of interactivity for several values of the beta function.

\begin{tabular}{cccc}
\hline $\mathrm{B}(\mathrm{a}, \mathrm{b})$ & $\mathrm{B}(4,3)$ & $\mathrm{B}(3,2)$ & $\mathrm{B}(2,1)$ \\
\hline$I_{\omega}(U, \Gamma)$ & 0.167502 & 0.159894 & 0.138927 \\
\hline $\mathrm{B}(\mathrm{a}, \mathrm{b})$ & $\mathrm{B}(5,3)$ & $\mathrm{B}(4,2)$ & $\mathrm{B}(3,1)$ \\
\hline$I_{\omega}(U, \Gamma)$ & 0.124546 & 0.107462 & 0.0760307 \\
\hline $\mathrm{B}(\mathrm{a}, \mathrm{b})$ & $\mathrm{B}(10,5)$ & $\mathrm{B}(5,1)$ & $\mathrm{B}(1000,50)$ \\
\hline$I_{\omega}(U, \Gamma)$ & 0.0876551 & 0.0332298 & 0.00147537 \\
\hline
\end{tabular}

Table 1 shows that the smaller is the difference between $a$ and $b$ in the beta function, the greater is the measure of interactivity between $U$ and $\Gamma$. Moreover, when the difference between $a$ and $b$ is constant, the measure of interactivity increases as $a$ grows. 
Figure 2 shows the graphical representation of the fuzzy plasma viral load population given by (6.4), that is, the fuzzy plasma viral load population when $U$ and $\Gamma$ are interactive fuzzy numbers, and its expected solution via (4.1) with $B(5,3)$.

By the other hand, Figure 3 shows the fuzzy plasma viral load population if we consider $U$ and $\Gamma$ non-interactive parameters. In this case, we consider the fuzzy numbers $U=(2 ; 3 ; 9)$ and $\Gamma=(0.08 ; 0.5 ; 1)$ as in $[8]$.

Herz et al. [8] estimates that the transition phase will occur between 1 and 2 days. More precisely, virus load starts to fall exponentially around 30 to 40 hours (approximately 1.2 to 1.6 days) after initiating treatment. This time span is a combination of the shoulder phase, a pharmacological delay of the drug and the intracellular phase of the virus life-cycle [3]. Furthermore, with the samples currently available, it is not possible to reliably distinguish the smoothness of the transition phase for different values of $u$ and with sufficient precision [8].

The same occurs in our model, which, despite using fuzzy parameters, does not present any distinction in the transition phase. The fact can be seen in Figure 2, where a tapering appears. This is caused by the effects of pharmacological delay, intracellular delay, and free virus decay during the transition phase. It is important to highlight that, in the case where the parameters are not interactive as in Figure 3, this distinction of smoothness occurs, contradicting the results of the experiments mentioned above.

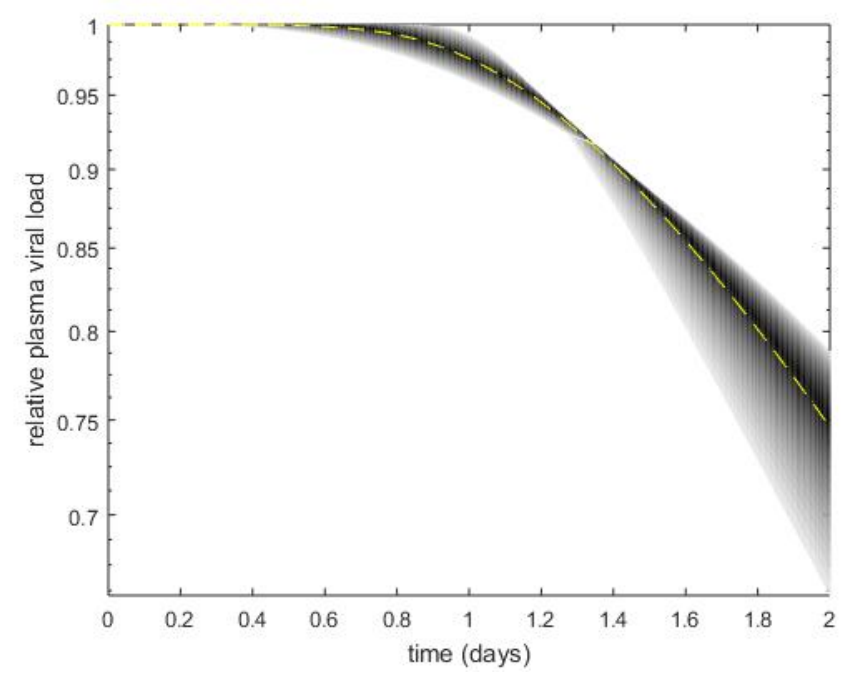

Figure 2: Graphical representation of the fuzzy plasma viral load population given by (6.4). The dashed-point curve is the expected solution of (6.4) with $B(5,3)$ (via (4.1)). The gray lines represent the $\alpha$-cuts of the $v_{t}(\tau, u)$. The endpoints for $\alpha$, varying from 0 to 1 , are represented respectively by the gray-scale lines varying from white to black. 


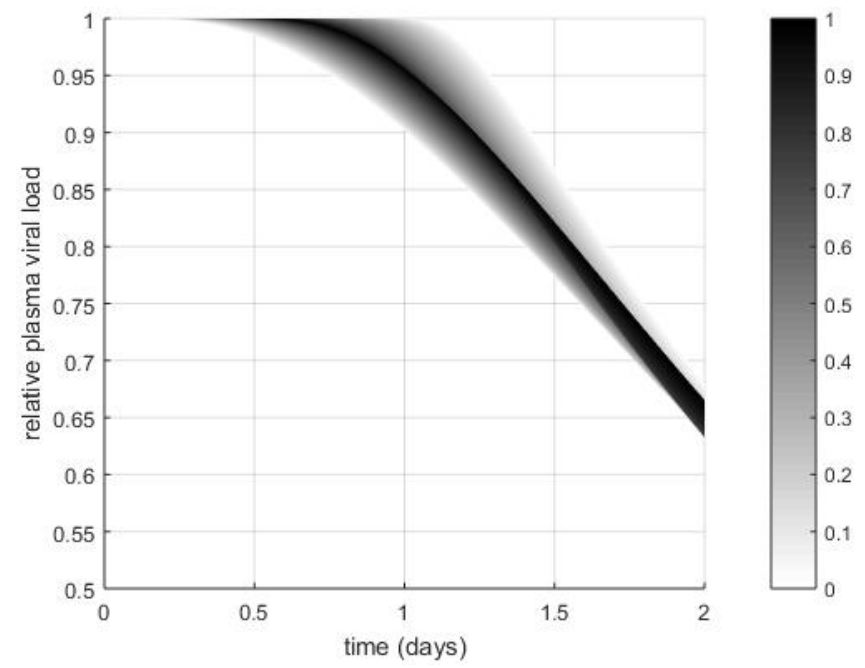

Figure 3: Graphical representation of the fuzzy plasma viral load population with non-interactive parameters. The gray lines represent the $\alpha$-cuts of the $v_{t}(\tau, u)$. The endpoints for $\alpha$, varying from 0 to 1 , are represented respectively by the gray-scale lines varying from white to black.

- The influence of interactivity between $U$ and $\Gamma$ on defuzzified solution.

The initial decline of the plasma virus in (6.4) is slow because the infectious virus that was produced before the start of the treatment continues to infect the cells. As one can see in Figure 4, different parameters in the beta distribution does not cause significant changes in the defuzzified curves.

In Figure 5 we compare the defuzzified solution of (6.4) with the several crisp solutions obtained by (5.5). This curve $c(t)=E_{\omega}\left(\left(v_{t}\right)_{J}(U, \Gamma)\right)$ does not coincide with the deterministic solution of (5.5) for fixed $u$ and $\tau$. The deterministic model excludes the uncertainty of the parameters at the beginning of the process, since we consider that those parameters are crisp.

By the other hand, when we consider the parameters to be fuzzy numbers, the uncertainties incorporated on the fuzzy model evolve through time and the defuzzification occurs at the instant of interest, thus obtaining a crisp curve. Figure 5 shows that the crisp curves differ mainly at the end of the shoulder phase, but even when the parameters vary, the differences are small. Besides that, the curve $c(t)$ leads to a smooth curve whereas the crisp curve leads to a sharp edge of the shoulder.

\section{CONCLUSIONS}

Herz et al. [8] have shown that intracellular delay may affect the magnitude of the range of HIV decline, this represents variability or uncertainty in response to protease inhibitor therapy. The 


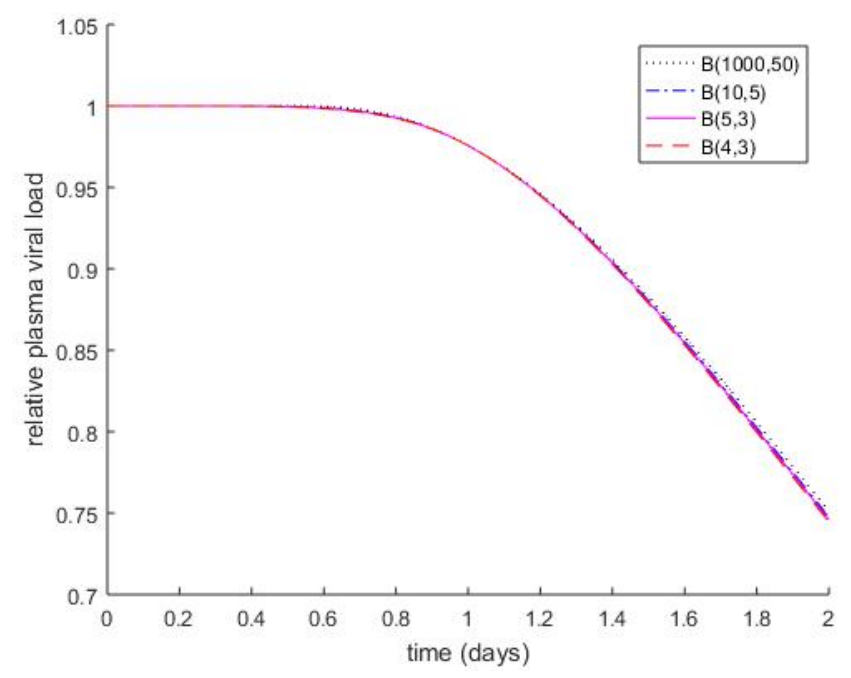

Figure 4: Expected curves of (6.4), $c(t)=E_{\omega}\left(\left(v_{t}\right)_{J}(U, \Gamma)\right)$, generated by (4.1) with interactive fuzzy numbers $\Gamma$ and $U$ given, respectively, by (6.2) and (6.3), and considering different beta distributions for $\alpha$.

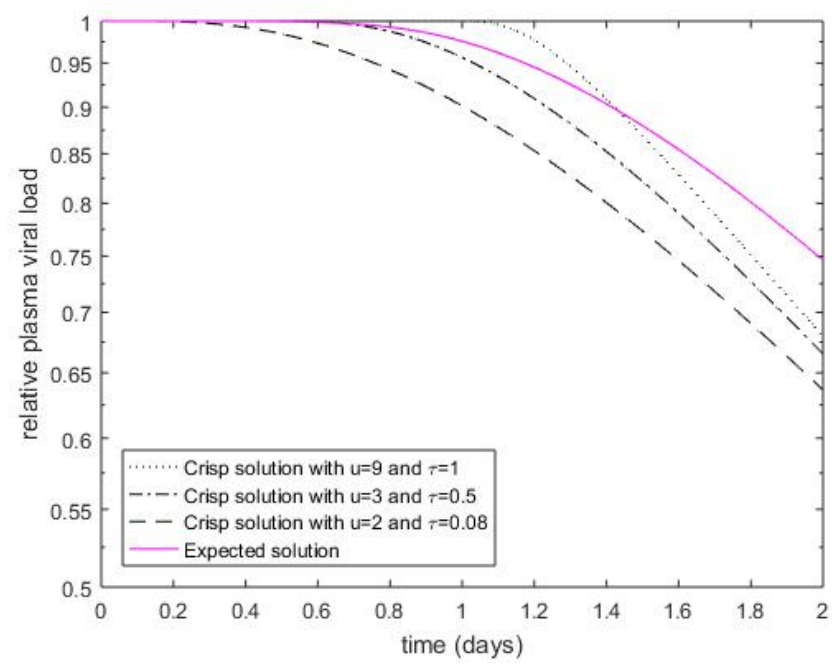

Figure 5: Deterministic solution of the plasma viral load population for various values of $u$ and $\tau$, and defuzzified solution of (6.4), $c(t)=E_{\omega}\left(\left(v_{t}\right)_{J}(U, \Gamma)\right)$, with interactive fuzzy numbers $\Gamma$ and $U$ given, respectively, by (6.2) and (6.3), and considering $B(5,3)$. 


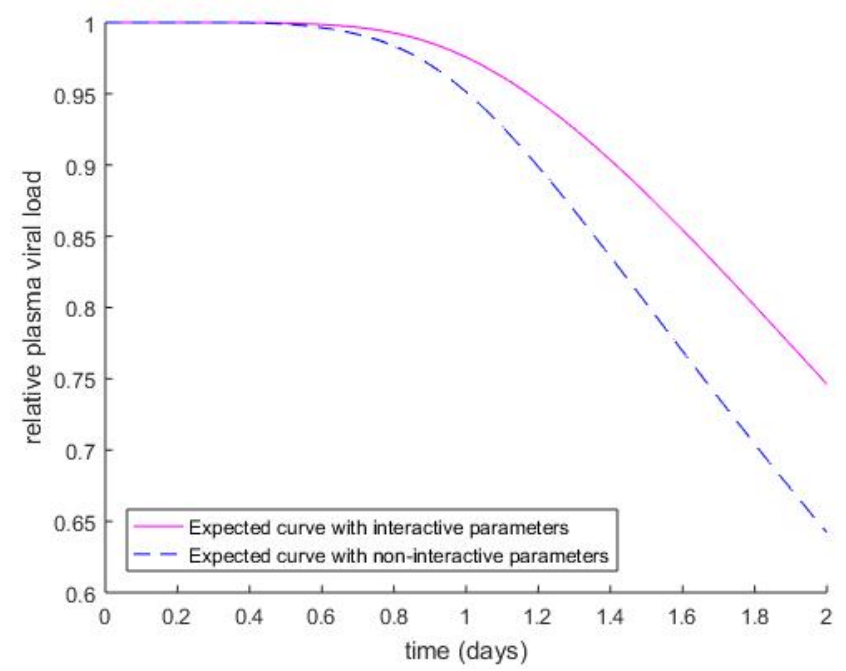

Figure 6: Expectation of the plasma viral load population for interactive and non-interactive fuzzy numbers $\Gamma$ and $U$, and considering $B(5,3)$.

adoption of delay via interactive fuzzy numbers has held good results in the study HIV dynamics under treatment.

We concluded that different parameters into the beta distribution led us similar defuzzified curves. Additionally, the measure of interactivity increases as the parameter $a$ (in beta function) increases. Besides that, we presented the fuzzy plasma viral load population with interactive and non-interactive parameters.

The data available at the moment do not allow the distinction of the transition phase in solutions with different parameter values [8]. Likewise, when considering interactive fuzzy parameters, modeled by hyperbolic fuzzy numbers, we did not observe a distinction between the effects of intracellular delay and virus deterioration free during the transition phase. This behavior, which is characteristic of the phenomenon, can be observed in the interactive solution curve (Figure 2) through the tapering that appears in the solution. In short, in deterministic models, uncertainties are excluded early in the process, whereas, in the presented study, the uncertainties are carried out through the process and, only at the end, those uncertainties are eliminated at the instant of interest.

\section{REFERENCES}

[1] L.C. Barros, R.C. Bassanezi \& W.A. Lodwick. "A First Course in Fuzzy Logic, Fuzzy Dynamical Systems, and Biomathematics", volume 347 of Studies in Fuzziness and Soft Computing. Springer, 1 ed. (2017).

[2] O.A.d. Barros. "Estimação dos parâmetros da distribuição beta bivariada: aplicações em severidade 
de doenças em plantas”. Master's thesis, Universidade de São Paulo (2015).

[3] S. Bonhoeffer, R.M. May, G.M. Shaw \& M.A. Nowak. Virus dynamics and drug therapy. Proceedings of the National Academy of Sciences, 94(13) (1997), 6971-6976.

[4] V. Cabral \& L.C. Barros. Fuzzy differential equation with completely correlated parameters. Fuzzy Sets and Systems, 265 (2015), 86-98.

[5] V.M. Cabral, R.A.C. Prata \& L.C. Barros. $f$-correlated fuzzy numbers applied to HIV model with protease inhibitor therapy. Mathware \& soft computing, 22(1), 46-51.

[6] C. Carlsson, R. Fullér et al. Additions of completely correlated fuzzy numbers. In "Fuzzy Systems, 2004. Proceedings. 2004 IEEE International Conference on”, volume 1. IEEE (2004), pp. 535-539.

[7] R. Fullér \& P. Majlender. On interactive fuzzy numbers. Fuzzy Sets and Systems, 143(3) (2004), 355369.

[8] A. Herz, S. Bonhoeffer, R.M. Anderson, R.M. May \& M.A. Nowak. Viral dynamics in vivo: limitations on estimates of intracellular delay and virus decay. Proceedings of the National Academy of Sciences, 93(14) (1996), 7247-7251.

[9] R.M. Jafelice, L. Barros \& R. Bassanezi. Study of the dynamics of HIV under treatment considering fuzzy delay. Computational and Applied Mathematics, 33(1) (2014), 45-61.

[10] E. Massad, N.R.S. Ortega, L.C. de Barros \& C.J. Struchiner. "Fuzzy logic in action: applications in epidemiology and beyond", volume 232. Springer Science \& Business Media (2009).

[11] J.E. Mittler, B. Sulzer, A.U. Neumann \& A.S. Perelson. Influence of delayed viral production on viral dynamics in HIV-1 infected patients. Mathematical biosciences, 152(2) (1998), 143-163.

[12] A.S. Perelson, A.U. Neumann, M. Markowitz, J.M. Leonard \& D.D. Ho. HIV-1 dynamics in vivo: virion clearance rate, infected cell life-span, and viral generation time. Science, 271(5255) (1996), $1582-1586$.

[13] F. Santo Pedro, L.C. de Barros \& E. Esmi. Population growth model via interactive fuzzy differential equation. Information Sciences, 481 (2019), 160-173. 